\title{
Relationship between inflammatory parameters and cardiovascular and lifestyle factors in the Mugello study oldest old
}

\author{
Raffaello Molino Lova ${ }^{1}$, Cristina Vassalle*,2, Elisabetta Masini ${ }^{1}$, Silvia Del Ry ${ }^{3}$, Manuela \\ Cabiati $^{3}$, Guido Pasquini ${ }^{1}$ \& Claudio Macchi ${ }^{1}$ \\ ${ }^{1}$ Don Gnocchi Foundation, IRCCS, Florence, Italy \\ ${ }^{2}$ Fondazione CNR-Regione Toscana G Monasterio, Pisa, Italy \\ ${ }^{3}$ Italian National Research Council, Istituto di Fisiologia Clinica, Pisa, Italy \\ *Author for correspondence: Tel.: +39 050 3153525; Fax: +39 050 3152166; cristina.vassalle@ftgm.it
}

\begin{abstract}
Aim: To explore possible inter-relationships of various biomarkers of inflammation and lifestyle and other cardiovascular risk factors (age, gender, smoking history, hypertension, Type 2 diabetes, dyslipidemia, alteration of circadian rhythms, body mass index, calf circumference, thigh circumference, abdominal circumference) in the Mugello study oldest old. Methods: In 399 noninstitutionalized nonagenarians (291 women), whole blood cells, mean platelet volume, C-reactive protein, uric acid, gamma-glutamyl transferase were assessed. Results: Aging resulted as the only independent determinant for uric acid $(<0.05)$, and abdominal circumference for C-reactive protein. Female gender $(<0.01)$, and thigh circumference $(<0.05)$ remained as determinants for mean platelet volume, age $(<0.01)$, and male gender $(<0.01)$ for gamma-glutamyl transferase, and Type 2 diabetes $(\leq 0.01)$ and alteration of circadian rhythms $(<0.05)$ for whole blood cells. Conclusion: Several inflammatory parameters remain associated with adverse lifestyle and cardiovascular risk factors even among nonagenarians.
\end{abstract}

\section{Graphical Abstract:}

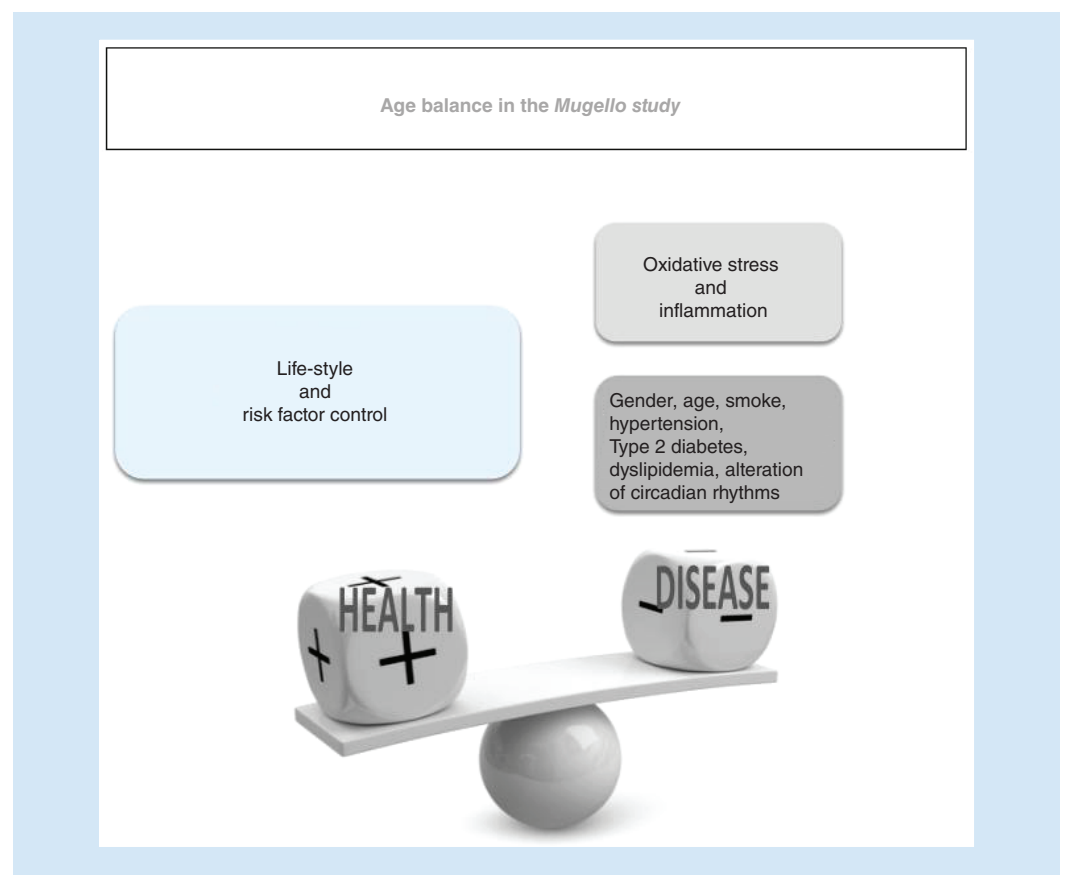

First draft submitted: 14 December 2017; Accepted for publication: 5 July 2018; Published online:

11 September 2018

Future Medicine 
Keywords: cardiovascular risk factors $\bullet$ inflammation $\bullet$ lifestyle $\bullet$ nonagenarians $\bullet$ oldest old $\bullet$ oxidative stress

Various lifestyle factors, including overweight, blood pressure and exercise, characterized by a certain grade of inflammation and oxidative stress, are related to mortality and longevity [1]. Conversely, different oxidative and inflammatory parameters have been associated to aging processes and chronic diseases. Specifically, a chronic, low-grade oxidative stress and inflammatory status likely characterized the onset and development of different age-related conditions, including cardiovascular (CV) disease [2]. In particular, uric acid (UA), a potent antioxidant, majorly responsible for antioxidant blood power, has been correlated with CV risk and events at higher levels [3]. C-reactive protein (CRP), an acute phase protein, has been found to be associated with disability, CV disease and mortality [4]. Various parameters obtained by hemochrome analysis, among which the white blood cells (WBC), and mean platelet volume (MPV), could be used as simple systemic inflammatory biomarkers [5-8]. Specifically, WBC has been identified as a predictor of CV risk and mortality $[5,6]$. Interestingly, MPV, a marker of platelet function, can be considered a potential link between inflammation and thrombosis and it has recently emerged as an effective marker of cardiometabolic risk [7,8]. Moreover, also GGT is associated with the development of $\mathrm{CV}$ disease and related risk factors, including diabetes, hypertension, dyslipidemia and retains pro-oxidant and pro-inflammatory properties [9,10].

Nonetheless, the significance of these oxidative stress and inflammatory biomarkers in very old cohorts is generally scarce, also considering that the information and reference values available for biomarkers commonly used in clinical routine may change with aging. Thus, studies focused on the relationship between oxidative stress and inflammatory biomarkers and lifestyle factors appear relevant to identify new potential interventional targets and improve our limited knowledge of the underlying pathophysiology in the oldest old population.

The Mugello study was conducted in the Mugello area, north eastward of Florence, Tuscany, Italy, which holds a high percentage of oldest old subjects in comparison to other Italian territories [11-13]. In this extraordinary population cohort, which included a large amount of subjects over 90 years, we assessed the distribution of the above reported and most commonly used biomarkers (CRP, UA, MPV, GGT, WBC), evaluating if their levels are modulated by gender, age, anthropometric measures, $\mathrm{CV}$ risk factors and lifestyle habits.

\section{Materials \& methods}

\section{Participants}

Results presented are based on data from the Mugello study, an epidemiologic, retrospective, single-center study on a large number of oldest old living in the Mugello area, whose details have been previously described [11]. In this survey, subjects have been evaluated for various health issues, including presence and extent of diseases, aspects related to nutritional status and routine hematology and biochemistry, and data collected in the Mugello Study database. Participation rate was high among oldest old epidemiologic studies, corresponding to 69\%.

Smoking was considered as a dichotomic variable, 'never smoked' versus 'current or former smoker'. Circadian rhythm alteration $(\mathrm{sCR})$ was defined according to presence of insomnia, irregular sleep-wake pattern, advanced sleep phase syndrome. Comorbid conditions, such as diabetes, hypertension, dyslipidemia - among others, were also considered as dichotomy variables and ascertained according to pre-established algorithms that combined information gathered from medical history, medical records, clinical examination and the blood and instrumental tests included in the Mugello study protocol [11].

The Mugello study protocol complied with the principles of the Declaration of Helsinki on clinical research involving human beings, and it was approved by the Institutional Review Board. Participants, or their proxies, signed the informed consent form to be included in the study and to undergo blood withdrawal. Among the Mugello Study participants, 399 subjects (291 women, mean \pm SD age $93.1 \pm 3.2$ years), had complete data to be included in this analysis.

\section{Anthropometric measurements}

Weight, ulna length, mid-upper arm circumference (MUAC), and calf circumference (CC) were measured as we previously described according to the standard procedures [12]. Weight was measured using a portable scale, and ulna length, MUAC and CC were measured using a nonstretchable fiberglass insertion tape. MUAC was measured halfway between the acromion and the olecranon process. The ulna length was measured between the olecranon and the midpoint of the styloid process; ulna length was used to estimate the subject height according to the MUST (BAPEN). Body mass index (BMI) was calculated from weight (W), in kilograms, and height $(\mathrm{H})$, in meters, 
according to the formula: $\mathrm{W} /(\mathrm{H})^{2}$. CC was measured by wrapping the tape around the widest part of the calf. MUAC and CC values were classified according to the following cut-offs: 21 and $22 \mathrm{~cm}$ for MUAC, and $31 \mathrm{~cm}$ for $\mathrm{CC}$ [12].

\section{Biochemical variables}

UA serum levels were analyzed using enzymatic-colorimetric methods (Roche Diagnostics GmbH, Mannheim, Germany) [13]. The lower LODs were $0.2 \mathrm{mg} / \mathrm{dl}$, range $0.2-25.0$. Intra- and interassay variation coefficients were 0.5 and $1.7 \%$, respectively.

High-sensitivity CRP was measured in duplicate using an ELISA and colorimetric competitive immunoassay that used purified protein and polyclonal anti-CRP antibodies. Values of white blood count and mean platelet volume (plasma-EDTA; XT 1800 SYSMEX DASIT, Japan), GGT (serum), and UA (serum) and CRP (serum) (DXC 600 Beckman Coulter, CA, USA) were measured.

\section{Statistical analysis}

Data are reported as mean \pm standard deviation or as a percentage. Statistical analysis performed included Student's t-test, $\chi^{2}$ test for continuous and categorical variables, respectively.

Continuous variables showing a markedly skewed distribution (CRP, GGT) were log-transformed before being entered into calculations. Log-transformed values were then back-transformed for data presentation. Categorical or continuous variables and independently associated with the dependent variable with a p-value $<0.05$ at the univariate analysis, entered in a multivariate regression model.

Analyses were performed using the statistical package Statview, version 5.0.1 (SAS Institute, Abacus Concept, Inc., CA, USA).

A p-value $\leq 0.05$ was considered statistically significant.

\section{Results}

\section{Characteristic of subjects}

The anthropometric and clinical characteristics of the 399 nonagenarian subjects in the overall population and according to gender are reported in Table 1. Males had higher AC and CC, and a higher risk for smoking history $(\mathrm{p}<0.001)$ than women (Table 1$)$. Instead, women were older than men $(\leq 0.01)$, and hypertension $(\mathrm{p}<0.01)$, physical inactivity $(<0.001)$ and the metabolic syndrome $(\mathrm{Met} S ; \leq 0.001)$ appeared to be more frequent in women than in men (Table 1). Among biochemical parameters, MPV was significantly higher and GGT was lower in females with respect to males (Table 1). No significant differences were observed according to the gender for the other anthropometric parameters and lifestyle factors considered (Table 1).

\section{Oxidative/inflammatory biomarker distribution \& correlation}

Considering that the information and reference values available for biomarkers commonly used in clinical routine changes with aging, we had evaluated the distribution of GGT, UA, CRP, MPV and WBC in the nonagenarian subjects enrolled in this study to verify their distribution against reference values used for clinical routine in our laboratory (Figure 1, panel A-E, respectively).

In this context, we found that 14,28,21, 9, and 5\% of Mugello oldest old subjects presented values higher than the high reference values utilized in our laboratory and corresponding respectively to $<38$ IU $/ 1$ for females and $<55 \mathrm{IU} / \mathrm{l}$ for males for GGT, $<6 \mathrm{mg} / \mathrm{dl}$ for females and $<7.2 \mathrm{mg} / \mathrm{dl}$ for males for UA, $<1 \mathrm{mg} / \mathrm{dl}$ for CRP, $<12 \mathrm{fl}$ for MPV and $10^{*} 10^{9} / 1$ for WBC (Figure 1 ).

In Table 2, the coefficients of correlation and the p-values obtained by the simple regression analysis among the biomarkers studied were reported. In particular, CRP levels correlated with all the other biomarkers (UA, MPV, GGT, WBC). Moreover, a significant direct association was also observed between UA and GGT.

\section{Biomarkers lifestyle determinants in oldest old}

In Table 3, we reported the multivariate analysis between all the parameters listed in Table 1 and the biomarkers analyzed. The only parameter associated with UA was aging $(0.11,2, \leq 0.05)$ (Table 3$)$.

Exploratory analyses revealed several significant independent associations between AC with CRP, gender and LC with MPV, as well as between age and male gender and GGT (Table 3). 
Table 1. Mugello study population characteristics.

\begin{tabular}{|c|c|c|c|c|}
\hline Characteristics & Overall population & Men & Women & p-value \\
\hline $\mathrm{n}$ & 399 & 108 & 291 & \\
\hline Age (years) & $93.1 \pm 3.2$ & $92.4 \pm 2.6$ & $93.3 \pm 3.3$ & $\leq 0.01$ \\
\hline Hypertension & $230(57)$ & $50(46)$ & $180(62)$ & $<0.01$ \\
\hline Diabetes & $58(14)$ & $13(12)$ & $45(15)$ & ns \\
\hline Dyslipidemia & $44(11)$ & $11(11)$ & $33(11)$ & ns \\
\hline MetS (NCEP-ATP III) & $108(27)$ & $18(16)$ & $90(31)$ & $\leq 0.001$ \\
\hline Alteration of circadian rhythms & $7(2)$ & $3(3)$ & $12(4)$ & ns \\
\hline Physical inactivity & & $44(41)$ & $170(60)$ & $<0.001$ \\
\hline Smoking history & $120(30)$ & $77(72)$ & $43(15)$ & $<0.001$ \\
\hline Mean blood pressure & $93 \pm 10$ & $92 \pm 10$ & $93 \pm 11$ & ns \\
\hline Body mass index $\left(\mathrm{kg} / \mathrm{m}^{2}\right)$ & $25 \pm 5$ & $25 \pm 3$ & $25 \pm 5$ & ns \\
\hline Abdominal circumference & $96 \pm 13$ & $100 \pm 12$ & $94 \pm 13$ & $<0.001$ \\
\hline Leg circumference & $45 \pm 7$ & $44 \pm 5$ & $45 \pm 8$ & ns \\
\hline Calf circumference & $32 \pm 5$ & $33 \pm 4$ & $31 \pm 5$ & $<0.001$ \\
\hline \multicolumn{5}{|l|}{ Laboratory biomarkers: } \\
\hline - Uric acid (mg/dl) & $5.7 \pm 1.8$ & $5.8 \pm 1.4$ & $5.6 \pm 1.9$ & ns \\
\hline - C-reactive protein $(\mathrm{mg} / \mathrm{dl})$ & $0.4(0.02-24.7)$ & $0.42(0.02-20.2)$ & $0.41(0.02-24.7)$ & ns \\
\hline - Mean platelet volume & $11 \pm 1.0$ & $11 \pm 1.0$ & $10 \pm 1.0$ & $<0.05$ \\
\hline $\begin{array}{l}\text { - Gamma-glutamyl transferase } \\
\text { (IU/I) }\end{array}$ & $17(3-220)$ & $19(6-162)$ & $16(3-220)$ & $<0.01$ \\
\hline - White blood count $\left(* 10^{9} / \mathrm{I}\right)$ & $6.4 \pm 1.9$ & $6.5 \pm 1.9$ & $6.3 \pm 1.9$ & ns \\
\hline
\end{tabular}

\begin{tabular}{|c|c|c|c|c|c|c|c|c|}
\hline & \multicolumn{2}{|c|}{ Uric acid } & \multicolumn{2}{|c|}{ Mean platelet volume } & \multicolumn{2}{|c|}{ Gamma-glutamyl transferase } & \multicolumn{2}{|c|}{ White blood count } \\
\hline & r-value & $p$-value & r-value & p-value & r-value & p-value & r-value & p-value \\
\hline $\begin{array}{l}\text { C-reactive } \\
\text { protein }\end{array}$ & 0.17 & $<0.01$ & -0.21 & $<0.001$ & 0.17 & $<0.001$ & 0.29 & $<0.001$ \\
\hline Uric acid & & & -0.02 & ns & 0.12 & $<0.05$ & 0.08 & ns \\
\hline $\begin{array}{l}\text { Mean platelet } \\
\text { volume }\end{array}$ & & & & & -0.07 & ns & -0.05 & ns \\
\hline $\begin{array}{l}\text { Gamma- } \\
\text { glutamyl } \\
\text { transferase }\end{array}$ & & & & & & & -0.03 & ns \\
\hline
\end{tabular}

Subjects with circadian rhythm alteration or T2D showed higher WBC with respect to those without these conditions (Table 3). Both factors remained independently related to WBC at the multivariate analysis (Table 3).

\section{Discussion}

Characteristic of subjects

Interestingly, in our oldest old population, we observed a low percentage of dyslipidemic subjects, with a very low percentage of subjects taking statins ( 7 and $11 \%$ in females and males, respectively). These percentages resulted significantly lower than those observed in younger age classes [14]. Nonetheless, these data confirmed the ones obtained in another Italian cohort of elderly subjects, indicating that both TotCH and TG resulted lower in oldest old with respect to elderly subjects [15]. All together these observations may suggest that lipid alteration might represent a strong determinant for mortality. In this context, specific lipid genotype (e.g., the apolipoprotein $\mathrm{E}$ [APOE] e2/3/4 polymorphism and the APOB rs1042034 [C/T]) and phenotype (e.g., HDL and LDL particle sizes) has been found strong predictors for longevity and life span $[16,17]$. 


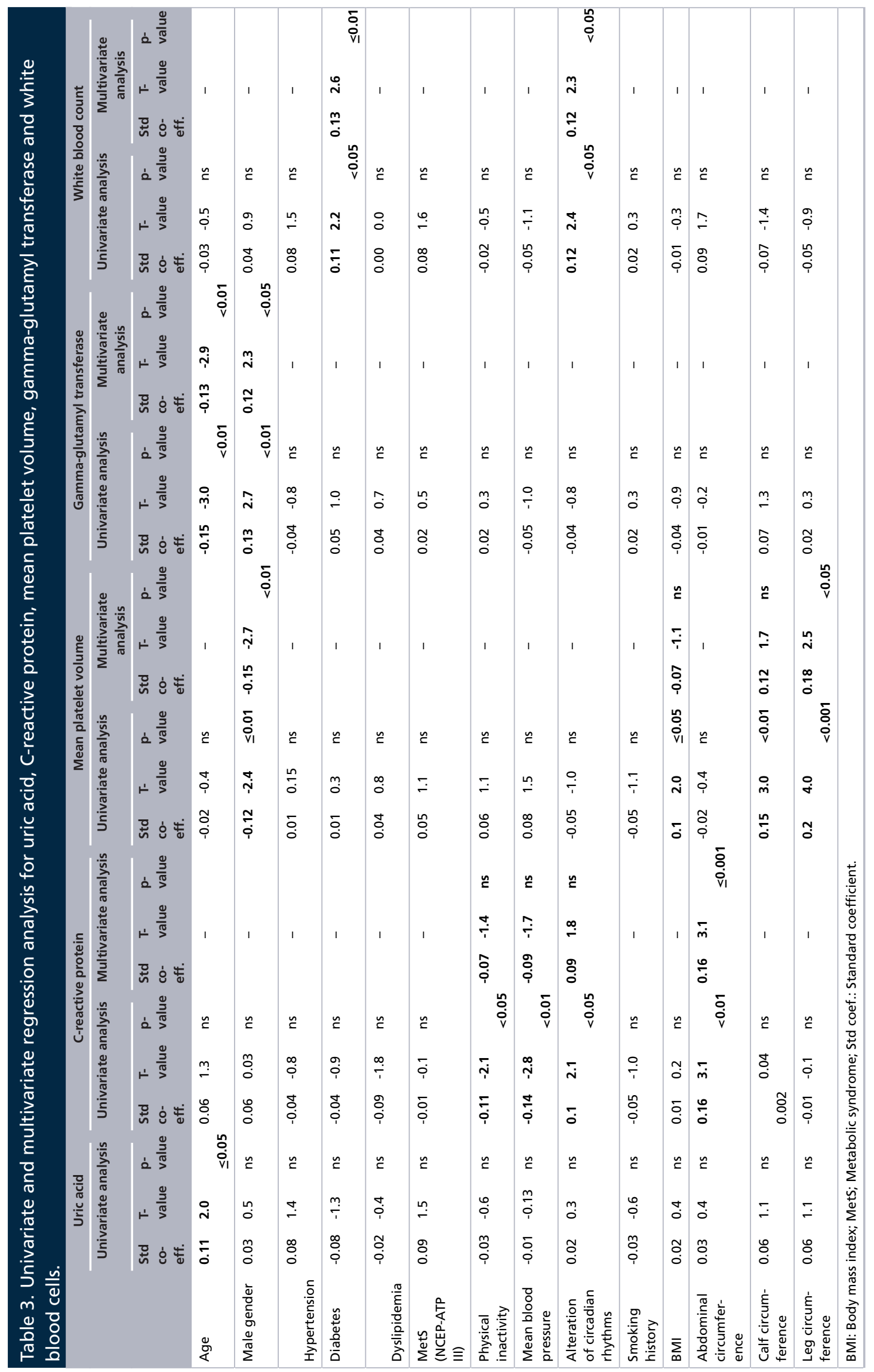




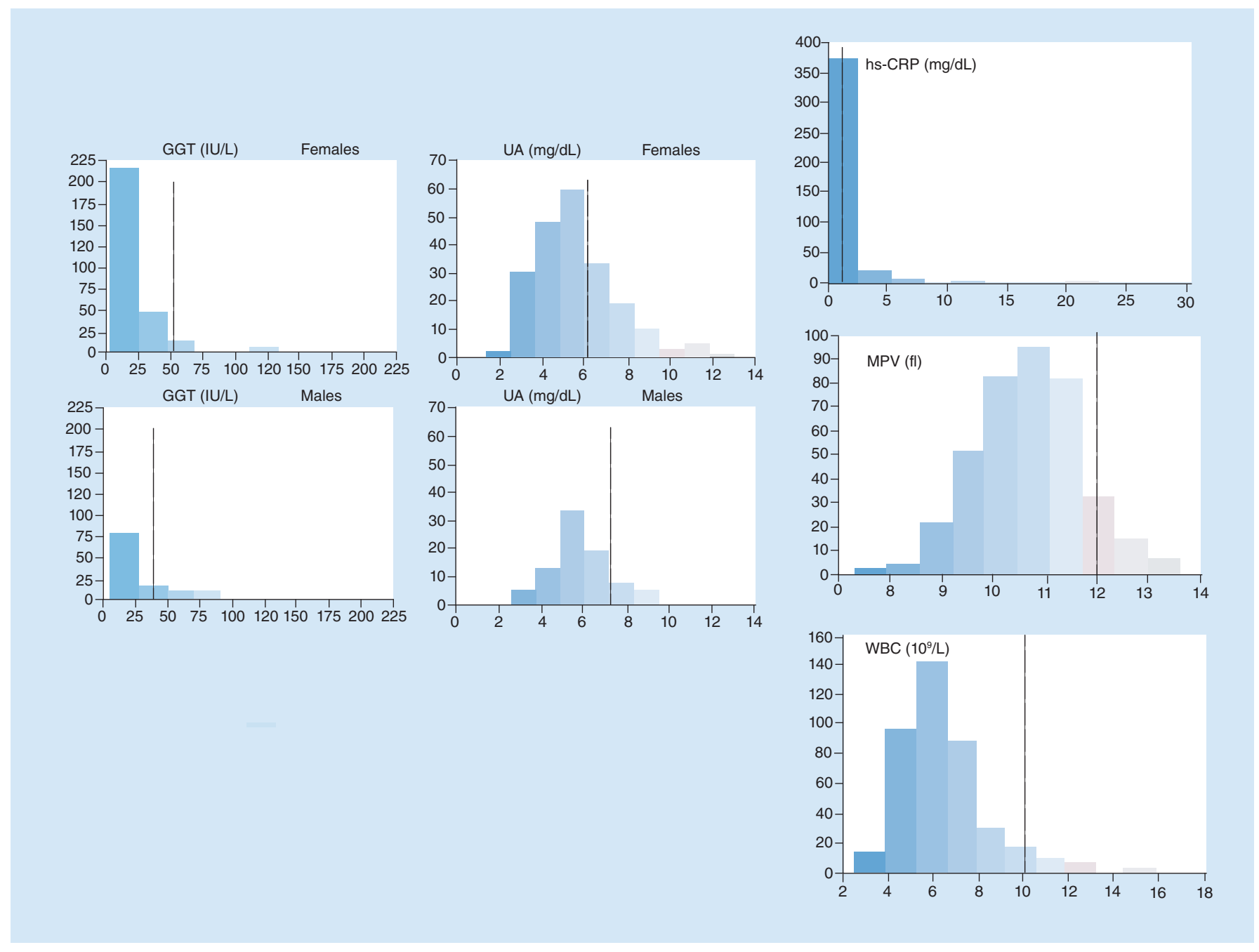

Figure 1. Distribution of gamma-glutamyl transferase, uric acid, C-reactive protein, mean platelet volume and white blood cells in the studied population.

In the Mugello cohort, also percentage of obesity did not result high, 11 and 14\% in male and female subjects. This fact may be beneficial in terms of survival. However, although longevity may be limited by the increased prevalence of obesity, some protective factors, as adiponectin, a serum protein expressed and secreted exclusively by adipose tissue, may retain a protective role against insulin resistance and atherosclerosis during aging [18], so this issue merits to be evaluated in the future study.

Interestingly, in a prospective study of healthy middle-age men, overweight and physical inactivity (in no-smoker) and smoking habit resulted as strong determinants of longevity (defined as reaching at least 85 years of age) [19]. In the Mugello study, the females were less active with respect to males (60 vs $42 \%$ the percentage of inactive subjects in the two genders, $\mathrm{p}<0.001$ ), while 30,9 and $1 \%$ in females and 30,26 and $2 \%$ in males performed light physical activity (2-4 h/week), moderate physical activity ( $>4 \mathrm{~h} /$ week or moderate [3-6 METs] physical activity $1-2 \mathrm{~h} /$ week) or intense ([ $>6 \mathrm{MET}]$ s physical activity several times a week), respectively. We did not observe any relationship between physical activity and the biomarkers considered. These findings are in agreement with the previous ones, showing that inflammatory components such as IL-1 $\beta$, TNF- $\alpha$, IL- 6 , IL-18 and CRP have inconsistent associations with different aspects of physical performance, obtained in older people (70-79 years) [20]. Moreover, we did not find any association between current or former smoking and risk factors considered, in agreement with previous data obtained in nonagenarians, which showed no role of smoking habit in systolic blood pressure or dyslipidemia, differently from the association of smoking habits with dyslipidemia in younger cohorts [21,22]. 


\section{Oxidative/inflammatory biomarker distribution \& correlation}

'Inflamm-aging', a chronic and low-grade inflammation that characterizes aging, appears to have important roles in late life [23,24]. In the Mugello oldest old, the percentage of subjects with levels higher than the high reference values varied according to the biomarker considered, from the lowest $5 \%$ for WBC $\left(12 * 10^{9} / 1\right)$, to $28 \%$ for UA $(<6 \mathrm{mg} / \mathrm{dl}$ for females and $<7.2 \mathrm{mg} / \mathrm{dl}$ for males). Moreover, the fact that we found that some biomarker increase with aging supports the hypothesis of 'inflamm-aging'.

\section{Uric acid}

We did not find any gender-related difference, whereas generally higher levels of UA are found in men than in women. Specifically, the lower UA plasma levels found in woman in younger age ranges has been attributed to the role of steroids in UA regulation, also called 'uricosuric effect', and to the possible urate depressing effect of estrogens in women [25]. However, results similar to our data were observed in the only study that evaluated a large Chinese population of older people (18,906 Chinese elderly aged 65 and older, which included a group of about 120 oldest old subject $>85$ years) [26].

Another interesting point is that although UA is not included between criteria for diagnosing metabolic syndrome (MetS), many studies have evidenced a positive association between UA and MetS in patients of various ages and ethnicities [3,27]. However, the lack of this relationship observed in our population is in agreement with the previous results, evidencing a higher MetS risk in the population ranging 65-74 years, but not in the oldest old subgroup ( 85 and over) [26]. The reason for this finding is unclear but could be explained by 'successful aging', characterized by a high level of functioning across several domains, against usual aging, in other words, functioning well but with a high risk of comorbidity and disability. Worthy of note, among the Mugello study participants, $32 \%$ were free from disabilities on the five-item basic ADL (bathing, dressing, toileting, transfer and feeding), 39\% were able to walk independently without any aid and 56\% showed Mini Mental State Examination raw scores $\geq 21$, which is generally considered as a cut-off value for cognitive impairment in older persons. Further, $22 \%$ lived alone needing, at most, some occasional support for heavy house chores.

\section{C-reactive protein}

CRP correlated with other different oxidative and inflammatory biomarkers, in agreement with previous ones obtained in younger age ranges [9,28]. Moreover, we found that AC was the only variable independently related to CRP. Interestingly, an association between increased CRP and obesity features has been previously found in younger age ranges, suggesting the possible use of CRP as additive biomarker to identify high-risk obese subjects also in the oldest old group [29].

\section{White blood count}

$\mathrm{T} 2 \mathrm{D}$ and circadian rhythm alteration resulted independently related to WBC at the multivariate analysis. Accordingly, a meta-analysis, which included data about the association between WBC count and T2D from 20 cross-sectional and prospective cohort observational studies (8647 T2D cases and 85,040 non cases) confirmed that raised WBC is associated with higher risk of T2D [30]. Moreover, we also observed a direct relationship between WBC and fast glicemia $(r=0.9, p \leq 0.05)$ and glycated hemoglobin $(r=0.22, p<0.001$; data not shown $)$. A reverse causality effect is also proposed because hyperglycemia itself may have an effect on WBC values, as antidiabetic drugs, such as rosiglitazone or acarbose, may lower WBC levels [31,32]. However, we did not observe this effect as subjects taking antidiabetic treatments presented higher levels of WBC with respect to those not taking these classes of drugs (6.9 \pm 2 vs $6.3 \pm 1.9 * 10^{9} / 1, \mathrm{p}<0.05$, data not shown).

\section{Sleep}

Sleep disturbance and desynchronization between endogenous rhythm and behavioral cycles are common among elderly people and represent important health issues for their relationship with an adverse metabolic profile, and a proinflammatory condition which can increase the risk of CV disease and early mortality. Although the mechanisms underlying these associations are not fully understood, data in younger age classes have evidenced the role of systemic inflammation through increases in WBC count, confirmed in our Mugello population [33,34]. If further studies will confirm this relationship also in the oldest old, approaches to lower disease risk associated with increased sleep-related inflammation should be evaluated in this particular population. 


\section{Mean platelet volume}

In younger classes of age, MPV resulted increased in obese patients, with concurrent reversal of such variable after weight loss [35]. This fact may be related to increase of circulating reticulated cells, which represent young, mRNA-rich platelets retaining a high pro-aggregating and hemostatic power [36]. However, we did not find any differences between MPV levels in obese and nonobese subjects ( $10.8 \pm 1.1 \mathrm{vs} 10.7 \pm 1 \mathrm{fl}, \mathrm{p}=\mathrm{ns}$, data not shown). Nonetheless, we observed an independent association between MPV and TC, which is an important nutritional and muscular mass index, especially in the elderly, which merits further deepening.

\section{Gamma-glutamyl transferase}

The relationship between aging and GGT that we observed in the Mugello cohort has been previously described in both genders of younger age, whereas males presented higher GGT levels than females [37,38]. Interestingly, GGT is emerging as a new strong and independent MetS determinant in middle age subjects [39,40]. However, our data did not support the association between GGT and MetS, thus GGT could not be an effective marker to identify this condition in nonagenarians.

\section{Study limitation}

One limitation can be related to the fact that some other important variables (e.g., socioeconomic status, social relationship and measurement of frailty index) might have a confounding effect on the results, but were not accounted in this analysis. As an example, it was not possible to assess what impact might have level of independence and quality of life, which were not, after all, in the focus of the present study. However, it is possible to recover all these parameters from the Mugello database, and undertake further studies which address this limitation in order to provide greater insights on this exceptionally elderly population.

\section{Clinical implication}

This study may inform about the levels of different risk and disease biomarkers still largely unknown in the oldest old subjects, so improving prevention and cure in this group of subjects, which represent the fastest growing section of the population by now. In this context, more knowledge about biological aspect of aging and the identification of critical biomarkers in healthy aging may also represent a useful tool for public health planning, allowing to promote a correct lifestyle and wellbeing across the life span, and prevent disabilities beyond the biological, psychological and social barriers that aging entails.

\section{Conclusion}

Several inflammatory parameters remain associated with adverse lifestyle and CV risk factors even among nonagenarians. Lifestyle modification and risk factor control could reduce inflammation and promote a healthy aging even at extreme old age.

\section{Summary points}

- The possible inter-relationships of various biomarkers of inflammation and lifestyle and other cardiovascular risk factors in oldest old of the Mugello study were evaluated.

- Several inflammatory parameters remain associated with adverse lifestyle and cardiovascular risk factors even among nonagenarians.

- Lifestyle and risk factor control could face inflammation and promote a healthy aging even at extreme old age.

\footnotetext{
Acknowledgements

The Mugello Study Working Group also includes: Roberta Boni, Chiara Castagnoli, Francesca Cecchi, Valentina Fabbri, Roberta Frandi, Annamaria Gori, Silvia Pancani, Anita Paperini, Lorenzo Razzolini, Nona Turcan, Debora Valecchi, Federica Vannetti.

Financial \& competing interests disclosure

The authors have no relevant affiliations or financial involvement with any organization or entity with a financial interest in or financial conflict with the subject matter or materials discussed in the manuscript. This includes employment, consultancies, honoraria, stock ownership or options, expert testimony, grants or patents received or pending, or royalties.

No writing assistance was utilized in the production of this manuscript.
} 


\section{Ethical conduct of research}

The authors state that they have obtained appropriate institutional review board approval or have followed the principles outlined in the Declaration of Helsinki for all human or animal experimental investigations. In addition, for investigations involving human subjects, informed consent has been obtained from the participants involved.

\section{References}

Papers of special note have been highlighted as: $\bullet$ of interest; $\bullet \bullet$ of considerable interest

1. Rea JN, Carvalho A, McNerlan SE, Alexander HD, Rea IM. Genes and lifestyle factors in BELFAST nonagenarians: nature, nurture and narrative. Biogerontology 16(5), 587-597 (2015).

2. Fougère B, Boulanger E, Nourhashémi F, Guyonnet S, Cesari M. Chronic inflammation: accelerator of biological aging. J. Gerontol. A. Biol. Sci. Med. Sci. 72(9), 1218-1225 (2016).

3. Vassalle C, Mazzone A, Sabatino L, Carpeggiani C. Uric acid for cardiovascular risk: Dr. Jekyll or Mr. Hide?” Diseases 4(1), 12 (2016).

4. Wang J, Tan GJ, Han LN, Bai YY, He M, Liu HB. Novel biomarkers for cardiovascular risk prediction. J. Geriatr. Cardiol. 14(2), 135-150 (2017).

\section{•• Overview of novel biomarkers to improve cardiovascular risk prediction.}

5. Barron E, Lara J, White M, Mathers JC. Blood-borne biomarkers of mortality risk: systematic review of cohort studies. PLoS ONE 10(6), e0127550 (2015).

6. Lowe GD. Circulating inflammatory markers and risks of cardiovascular and non-cardiovascular disease. J. Thromb. Haemost. 3(8), 1618-1627 (2005).

7. Chu SG, Becker RC, Berger PB et al. Mean platelet volume as a predictor of cardiovascular risk: a systematic review and meta-analysis. J. Thromb. Haemost. 8(1), 48-156 (2010)

- Evaluation of an emerging biomarker to improve cardiovascular risk estimation.

8. Zaccardi F, Rocca B, Pitocco D, Tanese L, Rizzi A, Ghirlanda G. Platelet mean volume, distribution width, and count in Type 2 diabetes, impaired fasting glucose, and metabolic syndrome: a meta-analysis. Diabetes Metab. Res. Rev. 31(4), 402-410(2015).

9. Vassalle C, Pratali L, Boni C, Mercuri A, Ndreu R. An oxidative stress score as a combined measure of the pro-oxidant and antioxidant counterparts in patients with coronary artery disease. Clin. Biochem. 41(14-15), 1162-1167 (2008).

10. Emdin M, Pompella A, Paolicchi A. Gamma-glutamyl transferase, atherosclerosis, and cardiovascular disease: triggering oxidative stress within the plaque. Circulation 112(14), 2078-2080 (2005).

11. Molino-Lova R, Sofi F, Pasquini G et al. The Mugello study, a survey of nonagenarians living in Tuscany: design, methods and participants' general characteristics. Eur. J. Intern. Med. 24(8), 745-749 (2013).

- Mugello study characteristics.

12. Bonaccorsi G, Santomauro F, Lorini C et al. Risk of malnutrition in a sample of nonagenarians: specific versus classic bioelectrical impedance vector analysis. Nutrition 32(2), 368-374 (2016).

13. Molino-Lova R, Sofi F, Pasquini G et al. Higher uric acid serum levels are associated with better muscle function in the oldest old: results from the Mugello study. Eur. J. Intern. Med. 41, 39-43 (2017).

14. Jacobs JM, Cohen A, Ein-Mor E, Stessman J. Cholesterol, statins, and longevity from age 70 to 90 years. J. Am. Med. Dir. Assoc. 14(12), 883-888 (2013).

15. Spazzafumo L, Olivieri F, Abbatecola AM et al. Remodelling of biological parameters during human ageing: evidence for complex regulation in longevity and in Type 2 diabetes. Age 35(2), 419-429 (2013).

16. Kulminski AM, Culminskaya I, Arbeev KG et al. The role of lipid-related genes, aging-related processes, and environment in healthspan. Aging Cell 12(2), 237-246 (2013).

17. Barzilai N, Atzmon G, Schechter C et al. Unique lipoprotein phenotype and genotype associated with exceptional longevity. JAMA 290(15), 2030-2040 (2003).

18. Atzmon G, Pollin TI, Crandall J et al. Adiponectin levels and genotype: a potential regulator of life span in humans. J. Gerontol. A. Biol. Sci. Med. Sci. 63(5), 447-453 (2008).

19. Heir T, Erikssen J, Sandvik L. Lifestyle and longevity among initially healthy middle-aged men: prospective cohort study. BMC Public Health 13, 831 (2013).

\section{•• Longitudinal study on the role of lifestyle variables for longevity.}

20. Hsu FC, Kritchevsky SB, Liu Y et al. Health ABC Study. Association between inflammatory components and physical function in the health, aging, and body composition study: a principal component analysis approach. J. Gerontol. A. Biol. Sci. Med. Sci. 64(5), 581-589 (2009).

21. Dong-Qing Z, Chang-Quan H, Yan-Ling Z, Bi-Rong D, Qing-Xiu L. Cigarette smoking is associated with increased diastolic blood pressure among Chinese nonagenarians/centenarians. Blood Press 23(3), 168-173 (2014). 
22. Yan-Ling Z, Dong-Qing Z, Chang-Quan H, Bi-Rong D. Cigarette smoking and its association with serum lipid/lipoprotein among Chinese nonagenarians/centenarians. Lipids Health Dis. 11, 94 (2012).

23. Michaud M, Balardy L, Moulis G et al. Proinflammatory cytokines, aging, and age-related diseases. J. Am. Med. Dir. Assoc. 14(12), 877-882 (2013).

24. Bell CL, Chen R, Masaki K et al. Late-life factors associated with healthy aging in older men. J. Am. Geriatr. Soc. 62(5), 880-888 (2014).

25. Adamopoulos D, Vlassopoulos C, Seitanides B, Contoyiannis P, Vassilopoulos P. The relationship of sex steroids to uric acid levels in plasma and urine. Acta Endocrinol. 85(1), 198-208 (1977).

26. Chen JH, Hsieh CH, Liu JS et al. The power of serum uric acid in predicting metabolic syndrome diminishes with age in an elderly Chinese population. J. Nutr. Health Aging 20(9), 912-917 (2016).

27. Caliceti C, Calabria D, Roda A, Cicero AFG. Fructose intake, serum uric acid, and cardiometabolic disorders: a critical review. Nutrients 9(4) pii:E395 (2017).

28. Vassalle C, Landi P, Boni C, Zucchelli G. Oxidative stress evaluated using an automated method for hydroperoxide estimation in patients with coronary artery disease. Clin. Chem. Lab. Med. 45(3), 367-371 (2007).

29. Mirhafez SR, Ebrahimi M, Saberi Karimian M et al. Serum high-sensitivity C-reactive protein as a biomarker in patients with metabolic syndrome: evidence-based study with 7284 subjects. Eur. J. Clin. Nutr. 70(11), 1298-1304 (2016).

30. Gkrania-Klotsas E, Ye Z, Cooper AJ et al. Differential white blood cell count and Type 2 diabetes: systematic review and meta-analysis of cross-sectional and prospective studies. PLoS ONE 5, e13405 (2010).

31. Van Wijk JP, Cabezas MC, Coll B et al. Effects of rosiglitazone on postprandial leukocytes and cytokines in Type 2 diabetes. Atherosclerosis 186(1), 152-159 (2006).

32. Hanefeld M, Schaper F, Koehler C et al. Effect of acarbose on postmeal mononuclear blood cell response in patients with early Type 2 diabetes: the AI(I)DA study. Horm. Metab. Res. 41(2), 132-136 (2009).

33. Boudjeltia KZ, Faraut B, Stenuit $\mathrm{P}$ et al. Sleep restriction increases white blood cells mainly neutrophil count, in young healthy men: a pilot study. Vasc. Health Risk Manag. 4(6), 1467-1470 (2008).

34. Sansanayudh N, Muntham D, Yamwong S, Sritara P, Akrawichien T, Thakkinstian A. The association between mean platelet volume and cardiovascular risk factors. Eur. J. Intern. Med. 30, 37-42 (2016).

35. Santilli F, Guagnano MT, Vazzana N, La Barba S, Davi G. Oxidative stress drivers and modulators in obesity and cardiovascular disease: from biomarkers to therapeutic approach. Curr. Med. Chem. 22(5), 582-595 (2015).

-• Overview of oxidative stress biomarkers in obesity and cardiovascular disease.

36. Chen BB, Shih TT, Hsu CY et al. Thigh muscle volume predicted by anthropometric measurements and correlated with physical function in the older adults. J. Nutr. Health Aging 15(6), 433-438 (2011).

37. Strømme JH, Rustad P, Steensland H, Theodorsen L, Urdal P. Reference intervals for eight enzymes in blood of adult females and males measured in accordance with the International Federation of Clinical Chemistry reference system at 37 degrees C: part of the Nordic Reference Interval Project. Scand. J. Clin. Lab. Invest. 64(4), 371-384 (2004).

38. Music M, Dervisevic A, Pepic E et al. Metabolic syndrome and serum liver enzymes level at patients with Type 2 diabetes mellitus. Med. Arch. 69(4), 251-255 (2015).

39. Onat A, Can G, Çakr H et al. Sex-specific predictors of metabolic syndrome independent of its components. J. Investig. Med. 63(6), 796-801 (2015).

40. Vigna L, Vassalle C, Tirelli AS et al. Gender related association between uric acid, homocysteine, gamma glutamyl transferase, inflammatory biomarkers, and metabolic syndrome in subjects affected by obesity. Biomark. Med. doi:10.2217/bmm-2017-0072 (2017). 